PROCEEDINGS OF THE

AMERICAN MATHEMATICAL SOCIETY

Volume 129, Number 12, Pages 3611-3618

S 0002-9939(01)05957-3

Article electronically published on April 16, 2001

\title{
WEAK INFINITE PRODUCTS OF BLASCHKE PRODUCTS
}

\author{
KEIJI IZUCHI
}

(Communicated by Albert Baernstein II)

\begin{abstract}
We study weak infinite products for sequences of Blaschke products. Using properties of these functions, $F_{\sigma}$-subsets of zero sets of functions in $H^{\infty}+C$ are studied. An affirmative answer is given to a problem on prime ideals of $H^{\infty}+C$ posed by Gorkin and Mortini.
\end{abstract}

\section{INTRODUCTION}

Let $D$ be the open unit disk in the complex plane. Let $L^{\infty}$ and $H^{\infty}$ be the usual Banach algebras on the unit circle $\partial D$. A closed subalgebra $B$ of $L^{\infty}$ containing $H^{\infty}$ properly is called a Douglas algebra. We denote by $M(B)$ the maximal ideal space of $B$. We may consider that $M(B)$ is a compact subset of $M\left(H^{\infty}\right)$. Also we may consider that $D \subset M\left(H^{\infty}\right)$, and the corona theorem says that $D$ is dense in $M\left(H^{\infty}\right)$ [1. The smallest Douglas algebra is $H^{\infty}+C$, where $C$ is the space of continuous functions on $\partial D$, and it is known that $M\left(H^{\infty}+C\right)=M\left(H^{\infty}\right) \backslash D$; see [3]. For a subset $E$ of $M\left(H^{\infty}+C\right)$, we denote by $\bar{E}$ and int $E$ the closure and the interior of $E$, respectively. For a function $f$ in $H^{\infty}+C$, we put

$Z(f)=\left\{\zeta \in M\left(H^{\infty}+C\right) ; f(\zeta)=0\right\}$ and $\{|f|<1\}=\left\{\zeta \in M\left(H^{\infty}+C\right) ;|f(\zeta)|<1\right\}$.

Similary, we define the set $\{f \neq 0\}$ in the space $M\left(H^{\infty}+C\right)$.

For a sequence $\left\{z_{j}\right\}_{j}$ in $D$ such that $\sum_{j=1}^{\infty}\left(1-\left|z_{j}\right|\right)<\infty$, there is the associated Blaschke product $b$ given by

$$
b(z)=\prod_{j=1}^{\infty} \frac{-\bar{z}_{j}}{\left|z_{j}\right|} \frac{z-z_{j}}{1-\bar{z}_{j} z}, \quad z \in D .
$$

It is known that $|b|=1$ on $M\left(L^{\infty}\right)$; see [10]. We denote by $\mathcal{P}(b)$ the set of sequences of positive integers $p=\left(p_{1}, p_{2}, \ldots\right)$ such that $\sum_{j=1}^{\infty} p_{j}\left(1-\left|z_{j}\right|\right)<\infty$ and $p_{j} \rightarrow \infty$ as $j \rightarrow \infty$. Then we have a family of Blaschke products

$$
b^{p}(z)=\prod_{j=1}^{\infty}\left(\frac{-\bar{z}_{j}}{\left|z_{j}\right|} \frac{z-z_{j}}{1-\bar{z}_{j} z}\right)^{p_{j}}, \quad p \in \mathcal{P}(b) .
$$

In [13, these Blaschke products were studied and called weak infinite powers of $b$. In this paper, we consider a slight generalization of it.

Received by the editors November 8, 1999 and, in revised form, April 20, 2000.

2000 Mathematics Subject Classification. Primary 46J15.

Supported by Grant-in-Aid for Scientific Research (No.10440039), Ministry of Education, Science and Culture. 
Let $B=\left(b_{1}, b_{2}, \ldots\right)$ be a sequence of infinite Blaschke products and $\left\{z_{k, j}\right\}_{j}$ be the zeros of $b_{k}$. Then there is a sequence of positive integers $N=\left(n_{1}, n_{2}, \ldots\right)$ such that

$$
\sum_{k=1}^{\infty} \sum_{j=n_{k}}^{\infty}\left(1-\left|z_{k, j}\right|\right)<\infty .
$$

We denote by $\mathcal{N}(B)$ the set of such sequences $N$. We put

$$
B_{N}=\prod_{k=1}^{\infty} \prod_{j=n_{k}}^{\infty} \frac{-\bar{z}_{k, j}}{\left|z_{k, j}\right|} \frac{z-z_{k, j}}{1-\bar{z}_{k, j} z}, \quad N \in \mathcal{N}(B),
$$

and we call $B_{N}$ a weak infinite product of Blaschke products $\left\{b_{k}\right\}_{k}$. In this paper, using them we study topological properties of $Z(f), f \in H^{\infty}+C$, and apply them to study prime ideals of $H^{\infty}+C$.

In Theorem 2.1, we prove that if $E$ is a $G_{\delta}$-subset of $M\left(H^{\infty}+C\right)$ such that $\overline{\bigcup_{k=1}^{\infty}\left\{\left|b_{k}\right|<1\right\}} \subset E$, then there exists $N \in \mathcal{N}(B)$ such that $\left\{\left|B_{N}\right|<1\right\} \subset E$. Applying Theorem 2.1, we get similar results obtained in 13. In Theorem 3.1, we prove that if $f \in H^{\infty}+C, f \neq 0$, and $E$ is an $F_{\sigma}$-subset of $M\left(H^{\infty}+C\right)$ satisfying $E \subset$ int $Z(f)$, then $\bar{E} \subset$ int $Z(f)$.

Applying Theorem 3.1, we solve a problem posed by Gorkin and Mortini [7] Q4]. The study of the structure of ideals in $H^{\infty}$ and $H^{\infty}+C$ is very attractive and many problems remain open (see [5, 6]). For a point $x \in M\left(H^{\infty}+C\right)$, we have two typical ideals. One is the maximal ideal $\left\{f \in H^{\infty}+C ; f(x)=0\right\}$ and the other is the ideal $J(x)$ which consists of functions in $H^{\infty}+C$ which vanish in a neighborhood of $x$. To investigate the structure of $H^{\infty}+C$, it is important to study the behavior of functions in $H^{\infty}+C$ in neighborhoods of $x$. In [7, the authors studied these ideals and posed the problem whether $J(x)$ is a prime ideal of $H^{\infty}+C$ or not. In Theorem 4.1, we prove that $J(x)$ is a prime ideal of $H^{\infty}+C$ for every $x \in M\left(H^{\infty}+C\right)$. This theorem will give some light on the study of prime ideals in $H^{\infty}$ and $H^{\infty}+C$.

\section{WEAK INFINITE PRODUCTS}

Let $B=\left(b_{1}, b_{2}, \ldots\right)$ be a sequence of infinite Blaschke products. For $N \in$ $\mathcal{N}(B), N=\left(n_{1}, n_{2}, \ldots\right)$, and a positive integer $k$, put

$$
b_{k, n_{k}}(z)=\prod_{j=n_{k}}^{\infty} \frac{-\bar{z}_{k, j}}{\left|z_{k, j}\right|} \frac{z-z_{k, j}}{1-\bar{z}_{k, j} z}, \quad z \in D .
$$

Then we have $B_{N}=\prod_{k=1}^{\infty} b_{k, n_{k}}$. Put

$$
\tilde{B}=\left(b_{1}, b_{1}, b_{2}, b_{1}, b_{2}, b_{3}, b_{1}, b_{2}, b_{3}, b_{4}, b_{1}, \ldots\right) .
$$

The sequence $\tilde{B}$ is called the associated sequence of $B$, and in the sequence $\tilde{B}$ each $b_{k}$ appears infinitely many times. By these definitions, it is not difficult to see the following.

Lemma 2.1. For each $N \in \mathcal{N}(\tilde{B})$, there exist $M \in \mathcal{N}(B), M=\left(m_{1}, m_{2}, \ldots\right)$, and $p_{k} \in \mathcal{P}\left(b_{k, m_{k}}\right)$ such that $\tilde{B}_{N}=\prod_{k=1}^{\infty} b_{k, m_{k}}^{p_{k}}$.

For a Blaschke product $b$, we denote by $S(b)$ the closed subset of $\partial D$ on which $b$ cannot be extended analytically. For a subset $E \subset L^{\infty}, H^{\infty}[E]$ is denoted by the Douglas algebra generated by $H^{\infty}$ and functions in $E$. In [13, Theorem 3.1], the 
author proved that $L^{\infty}=H^{\infty}\left[\overline{b^{p}} ; p \in \mathcal{P}(b)\right]$ if and only if $S(b)=\partial D$, where the bar denotes the complex conjugate.

Proposition 2.1. Let $B=\left(b_{1}, b_{2}, \ldots\right)$ be a sequence of infinite Blaschke products and $\tilde{B}$ the associated sequence of $B$. If $\bigcup_{k=1}^{\infty} S\left(b_{k}\right)$ is dense in $\partial D$, then $L^{\infty}=$ $H^{\infty}\left[\overline{\tilde{B}}_{N} ; N \in \mathcal{N}(\tilde{B})\right]$.

Proof. Fix $N_{0} \in \mathcal{N}(\tilde{B})$ and put $b=\tilde{B}_{N_{0}}$. By Lemma 2.1, there exist $M \in$ $\mathcal{N}(B), M=\left(m_{1}, m_{2}, \ldots\right)$, and $p_{k} \in \mathcal{P}\left(b_{k, m_{k}}\right)$ such that $b=\prod_{k=1}^{\infty} b_{k, m_{k}}^{p_{k}}$. Let $p \in \mathcal{P}(b)$. Then it is easy to see the existence of $N_{1} \in \mathcal{N}(\tilde{B})$ such that $\tilde{B}_{N_{1}}=b^{p}$. Hence

$$
\left\{b^{p} ; p \in \mathcal{P}(b)\right\} \subset\left\{\tilde{B}_{N} ; N \in \mathcal{N}(\tilde{B})\right\} .
$$

Since $\bigcup_{k=1}^{\infty} S\left(b_{k}\right)=\bigcup_{k=1}^{\infty} S\left(b_{k, m_{k}}\right) \subset S(b)$, by our assumption we have $S(b)=\partial D$. By the result mentioned above, we have $L^{\infty}=H^{\infty}\left[\overline{b^{p}} ; p \in \mathcal{P}(b)\right]$. Hence by (2.1), $L^{\infty}=H^{\infty}\left[\tilde{\tilde{B}}_{N} ; N \in \mathcal{N}(\tilde{B})\right\}$.

Theorem 2.1. Let $B=\left(b_{1}, b_{2}, \ldots\right)$ be a sequence of infinite Blaschke products. Let $E$ be a $G_{\delta}$-subset of $M\left(H^{\infty}+C\right)$ such that $\overline{\bigcup_{k=1}^{\infty}\left\{\left|b_{k}\right|<1\right\}} \subset E$. Then there exists $N \in \mathcal{N}(B)$ such that $\left\{\left|B_{N}\right|<1\right\} \subset E$.

Proof. The idea of the proof is the same as the one of [13, Theorem 2.4]. For the reader's convenience, we give the proof.

Since $E$ is a $G_{\delta^{-}}$-set, there is a sequence of closed subsets $\left\{K_{n}\right\}_{n}$ of $M\left(H^{\infty}+C\right)$ such that

$$
M\left(H^{\infty}+C\right) \backslash E=\bigcup_{n=1}^{\infty} K_{n} .
$$

By our assumption, there is an open subset $V_{n}$ of $M\left(H^{\infty}\right)$ such that

$$
K_{n} \subset V_{n} \text { and } \overline{\bigcup_{k=1}^{\infty}\left\{\left|b_{k}\right|<1\right\}} \cap \bar{V}_{n}=\emptyset .
$$

By the corona theorem,

$$
\bar{V}_{n} \backslash D=\overline{V_{n} \cap D} \backslash D .
$$

By (2.3),

$$
\left|b_{k}\right|=1 \text { on } \bar{V}_{n} \backslash D \text { for every } k \text { and } n .
$$

Let $\left\{\varepsilon_{k}\right\}_{k}$ be a sequence of positive numbers with $0<\varepsilon_{k}<1$ such that

$$
\prod_{k=1}^{\infty} \varepsilon_{k}>0
$$

By (2.4) and (2.5), for each positive integer $k$ there is a sufficiently large positive integer $n_{k}$ such that

$$
\left|b_{k, n_{k}}\right|>\varepsilon_{k} \quad \text { on } V_{n} \cap D \text { for every } n, 1 \leq n \leq k .
$$


Moreover, we may assume that $\sum_{k=1}^{\infty} \sum_{j=n_{k}}^{\infty}\left(1-\left|z_{k, j}\right|\right)<\infty$. Put $N=\left(n_{1}, n_{2}, \ldots\right)$. Then $N \in \mathcal{N}(B)$ and we have a Blaschke product $B_{N}=\prod_{k=1}^{\infty} b_{k, n_{k}}$. Fix $n$ arbitrary. By (2.7), for every $i \geq n$ we have

$$
\left|B_{N}\right| \geq\left(\prod_{k=1}^{i-1}\left|b_{k, n_{k}}\right|\right) \prod_{k=i}^{\infty} \varepsilon_{k} \quad \text { on } V_{n} \cap D .
$$

Then by (2.4) and (2.5), $\left|B_{N}\right| \geq \prod_{k=i}^{\infty} \varepsilon_{k}$ on $\bar{V}_{n} \backslash D$ for every $i \geq n$. Hence by (2.3) and $(2.6),\left|B_{N}\right|=1$ on $K_{n}$. Thus by $(2.2),\left|B_{N}\right|=1$ on $M\left(H^{\infty}+C\right) \backslash E$. Hence $\left\{\left|B_{N}\right|<1\right\} \subset E$.

Corollary 2.1. Let $B=\left(b_{1}, b_{2}, \ldots\right)$ be a sequence of infinite Blaschke products. Then

$$
\overline{\bigcup_{k=1}^{\infty}\left\{\left|b_{k}\right|<1\right\}}=\bigcap\left\{\overline{\left\{\left|B_{N}\right|<1\right\}} ; N \in \mathcal{N}(B)\right\} .
$$

Proof. It is easy to see that $\overline{\bigcup_{k=1}^{\infty}\left\{\left|b_{k}\right|<1\right\}} \subset \overline{\left\{\left|B_{N}\right|<1\right\}}$ for every $N \in \mathcal{N}(B)$. Let $x \in M\left(H^{\infty}+C\right) \backslash \overline{\bigcup_{k=1}^{\infty}\left\{\left|b_{k}\right|<1\right\}}$. Take an open subset $V$ of $M\left(H^{\infty}+C\right)$ such that $x \in V$ and $\bar{V} \cap \overline{\bigcup_{k=1}^{\infty}\left\{\left|b_{k}\right|<1\right\}}=\emptyset$. Then by Theorem 2.1, there exists $N \in \mathcal{N}(B)$ such that $\left|B_{N}\right|=1$ on $\bar{V}$. Hence $x \notin \overline{\left\{\left|B_{N}\right|<1\right\}}$.

Corollary 2.2. Let $B=\left(b_{1}, b_{2}, \ldots\right)$ be a sequence of infinite Blaschke products and $\tilde{B}$ the associated sequence of $B$. Let $E$ be a $G_{\delta}$-subset of $M\left(H^{\infty}+C\right)$ such that $\overline{\bigcup_{k=1}^{\infty}\left\{\left|b_{k}\right|<1\right\}} \subset E$. Then there is $N \in \mathcal{N}(\tilde{B})$ such that

$$
\overline{\bigcup_{k=1}^{\infty}\left\{\left|b_{k}\right|<1\right\}} \subset Z\left(\tilde{B}_{N}\right) \subset\left\{\left|\tilde{B}_{N}\right|<1\right\} \subset E .
$$

Proof. Put $\tilde{B}=\left(c_{1}, c_{2}, \ldots\right)$. Then by our assumption, $\overline{\bigcup_{k=1}^{\infty}\left\{\left|c_{k}\right|<1\right\}} \subset E$. By Theorem 2.1, there exists $N \in \mathcal{N}(\tilde{B})$ such that $\left\{\left|\tilde{B}_{N}\right|<1\right\} \subset E$. By Lemma 2.1, there exist $M \in \mathcal{N}(B), M=\left(m_{1}, m_{2}, \ldots\right)$, and $p_{k} \in \mathcal{P}\left(b_{k, m_{k}}\right)$ such that $\tilde{B}_{N}=\prod_{k=1}^{\infty} b_{k, m_{k}}^{p_{k}}$. By [13, Lemma 2.1], $\overline{\left\{\left|b_{k}\right|<1\right\}}=\overline{\left\{\left|b_{k, m_{k}}\right|<1\right\}} \subset Z\left(b_{k, m_{k}}^{p_{b}}\right)$. Hence we get

$$
\overline{\bigcup_{k=1}^{\infty}\left\{\left|b_{k}\right|<1\right\}} \subset Z\left(\tilde{B}_{N}\right) \subset\left\{\left|\tilde{B}_{N}\right|<1\right\} \subset E .
$$

By Corollary 2.2, we have the following.

Corollary 2.3. Let $B=\left(b_{1}, b_{2}, \ldots\right)$ be a sequence of infinite Blaschke products and $\tilde{B}$ the associated sequence of $B$. Then

$$
\overline{\bigcup_{k=1}^{\infty}\left\{\left|b_{k}\right|<1\right\}}=\bigcap\left\{Z\left(\tilde{B}_{N}\right) ; N \in \mathcal{N}(\tilde{B})\right\}=\bigcap\left\{\left\{\left|\tilde{B}_{N}\right|<1\right\} ; N \in \mathcal{N}(\tilde{B})\right\} .
$$

\section{3. $F_{\sigma}$-SETS}

For a point $x \in M\left(H^{\infty}\right)$, there is a unique probability measure $\mu_{x}$ on $M\left(L^{\infty}\right)$ such that $f(x)=\int_{M\left(L^{\infty}\right)} f d \mu_{x}$ for every $f \in H^{\infty}$; see [10. We denote by supp $\mu_{x}$ the closed support set of $\mu_{x}$. Let $b$ be a Blaschke product. Then $|b(x)|<1$ if and only if $b$ is not constant on $\operatorname{supp} \mu_{x}$. A sequence $\left\{z_{n}\right\}_{n}$ in $D$ is called interpolating if for every sequence of bounded complex numbers $\left\{a_{n}\right\}_{n}$ there exists $h \in H^{\infty}$ such 
that $h\left(z_{n}\right)=a_{n}$ for every $n$. A Blaschke product $b$ is called also interpolating if the sequence of zeros of $b$ in $D$ is interpolating. We denote by $G$ the set of all points $x$ in $M\left(H^{\infty}+C\right)$ such that $b(x)=0$ for some interpolating Blaschke product $b$. The structure of $G$ is well studied in [11]. For $x, y \in M\left(H^{\infty}\right)$, let $\rho(x, y)=$ $\sup \left\{|f(y)| ; f(x)=0,\|f\| \leq 1, f \in H^{\infty}\right\}$. Put $P(x)=\left\{\zeta \in M\left(H^{\infty}\right) ; \rho(x, \zeta)<\right.$ 1\}. Hoffman showed that $G=\bigcup\left\{P(x) ; P(x) \neq\{x\}, x \in M\left(H^{\infty}\right) \backslash D\right\}$, and if $P(x) \neq\{x\}$ there is a one-to-one continuous map $L_{x}$ from $D$ onto $P(x)$ such that $f \circ L_{x} \in H^{\infty}$ for every $f \in H^{\infty}$ and $L_{x}(0)=x$. Moreover if $f(x)=0$, then we can define order of zero of $f$ at $x$, ord $(f, x)$, as the order of zero of $f \circ L_{x}$ at 0 in $D$. When $P(x)=\{x\}$ and $f(x)=0$, we put $\operatorname{ord}(f, x)=\infty$. Hence $\operatorname{ord}(f, x)=\infty$ if and only if $f=0$ on $P(x)$. When $f \in J(x)$, we have $\operatorname{ord}(f, x)=\infty$.

For a function $f \in L^{\infty}$, let

$$
\tilde{f}(\zeta)=\int_{M\left(L^{\infty}\right)} f d \mu_{\zeta} \quad \text { for } \zeta \in M\left(H^{\infty}\right) .
$$

Then $\tilde{f}$ is a continuous function on $M\left(H^{\infty}\right)$; see [3, p. 375].

The following is the main theorem in this paper.

Theorem 3.1. Let $f \in H^{\infty}+C, f \neq 0$ and int $Z(f) \neq \emptyset$. Let $E$ be an $F_{\sigma}$-subset of $M\left(H^{\infty}+C\right)$ such that $E \subset$ int $Z(f)$. Then $\bar{E} \subset$ int $Z(f)$.

To prove our theorem, we use the following lemmas.

Lemma 3.1. Let $E$ be an $F_{\sigma}$-subset of $M\left(H^{\infty}+C\right)$ such that $E \cap M\left(L^{\infty}\right)=\emptyset$. Then $\bar{E} \cap M\left(L^{\infty}\right)=\emptyset$.

Proof. Since $E$ is an $F_{\sigma}$-set, it is not difficult to see the existence of a sequence of Blaschke products $\left\{b_{n}\right\}_{n}$ such that $E \subset \bigcup_{n=1}^{\infty}\left\{\left|b_{n}\right|<1\right\}$. Hence the assertion follows from 15 .

Lemma 3.2. Let $f \in H^{\infty}+C$ and $b$ a product of finitely many interpolating Blaschke products. If ord $(f, x)=\infty$ for every $x \in Z(b)$, then $\{|b|<1\} \subset Z(f)$.

The proof follows from [8, 9 .

Proof of Theorem 3.1. By our assumption,

$$
E \subset \operatorname{int} Z(f),
$$

and let $\left\{K_{n}\right\}_{n}$ be a sequence of compact subsets of $M\left(H^{\infty}+C\right)$ such that

$$
E=\bigcup_{n=1}^{\infty} K_{n}
$$

First, we prove the following case.

Case 1. $E \cap M\left(L^{\infty}\right)=\emptyset$.

Let $n$ be a fixed positive integer. Take $\zeta \in K_{n}$ arbitrary. When $\zeta \in G$, by (3.2) and (3.3) there exists an interpolating Blaschke product $q_{\zeta}$ such that $q_{\zeta}(\zeta)=0$ and $Z\left(q_{\zeta}\right) \subset$ int $Z(f)$. When $\zeta \notin G$, by [4] Corollary 3.2] there exists $\xi \in G \cap$ int $Z(f)$ such that

$$
\operatorname{supp} \mu_{\xi} \subset \operatorname{supp} \mu_{\zeta}
$$


Take an interpolating Blaschke product $q_{\zeta}$ such that $q_{\zeta}(\xi)=0$ and $Z\left(q_{\zeta}\right) \subset$ int $Z(f)$. By (3.4), $\left|q_{\zeta}(\zeta)\right|<1$. Hence $K_{n} \subset \bigcup_{\zeta \in K_{n}}\left\{\left|q_{\zeta}\right|<1\right\}$. Since $K_{n}$ is compact, there exist $\zeta_{1}, \zeta_{2}, \ldots, \zeta_{k}$ in $K_{n}$ such that

$$
K_{n} \subset\left\{\prod_{i=1}^{k}\left|q_{\zeta_{i}}\right|<1\right\} \text { and } Z\left(\prod_{i=1}^{k} q_{\zeta_{i}}\right) \subset \operatorname{int} Z(f) .
$$

Put $b_{n}=\prod_{i=1}^{k} q_{\zeta_{i}}$. Then by the above,

$$
K_{n} \subset\left\{\left|b_{n}\right|<1\right\}
$$

and

$$
Z\left(b_{n}\right) \subset \operatorname{int} Z(f) .
$$

We have $\operatorname{ord}(f, \zeta)=\infty$ for every $\zeta \in \operatorname{int} Z(f)$. Then by (3.6) and Lemma 3.2 we have $\left\{\left|b_{n}\right|<1\right\} \subset Z(f)$. Since $Z(f)$ is a $G_{\delta}$-set, we can apply Corollary 2.2. Put $B=\left(b_{1}, b_{2}, \ldots\right)$, and let $\tilde{B}$ be the associated sequence of $B$. Then by Corollary 2.2 , there exists $N \in \mathcal{N}(\tilde{B})$ such that

$$
\overline{\bigcup_{n=1}^{\infty}\left\{\left|b_{n}\right|<1\right\}} \subset Z\left(\tilde{B}_{N}\right) \subset\left\{\left|\tilde{B}_{N}\right|<1\right\} \subset Z(f) .
$$

Hence by (3.3) and (3.5), $\bar{E} \subset Z\left(\tilde{B}_{N}\right) \subset\left\{\left|\tilde{B}_{N}\right|<1\right\} \subset Z(f)$. Thus we get our assertion.

Next, we prove the following case.

Case 2. $E \cap M\left(L^{\infty}\right) \neq \emptyset$.

To prove our assertion, suppose that $\bar{E} \not \subset$ int $Z(f)$. Take a point $x_{0}$ satisfying

$$
x_{0} \in \bar{E} \quad \text { and } \quad x_{0} \notin \operatorname{int} Z(f) .
$$

Then two cases occur: $x_{0} \notin M\left(L^{\infty}\right)$ and $x_{0} \in M\left(L^{\infty}\right)$.

First suppose that $x_{0} \notin M\left(L^{\infty}\right)$. By Newman's theorem [14], there is a Blaschke product $\varphi$ such that $\varphi\left(x_{0}\right)=0$. By (3.7), we have

$$
x_{0} \in \overline{E \cap\{|\varphi|<1\}} \text {. }
$$

Since $E$ and $\{|\varphi|<1\}$ are $F_{\sigma}$-sets, $E \cap\{|\varphi|<1\}$ is an $F_{\sigma}$-set. Since

$$
(E \cap\{|\varphi|<1\}) \cap M\left(L^{\infty}\right)=\emptyset,
$$

we can apply Case 1. Hence $\overline{E \cap\{|\varphi|<1\}} \subset \operatorname{int} Z(f)$. By (3.8), $x_{0} \in \operatorname{int} Z(f)$ and this contradicts (3.7).

Now, suppose that $x_{0} \in M\left(L^{\infty}\right)$. Put

$$
S_{1}=\overline{M\left(L^{\infty}\right) \cap \operatorname{int} Z(f)} \quad \text { and } \quad S_{2}=\overline{M\left(L^{\infty}\right) \cap\{f \neq 0\}} .
$$

By (3.2), $M\left(L^{\infty}\right) \cap i n t Z(f) \neq \emptyset$. Since $M\left(L^{\infty}\right)$ is extremely disconnected [2, p. 18], $S_{1}$ and $S_{2}$ are non-empty open-closed subsets of $M\left(L^{\infty}\right)$, and

$$
S_{1} \cap S_{2}=\emptyset .
$$

Put

$$
E_{1}=E \cap\left\{\tilde{\chi}_{S_{1}}<1\right\} \quad \text { and } \quad E_{2}=E \cap\left\{\tilde{\chi}_{S_{1}}=1\right\} .
$$


Then $E_{1}$ is an $F_{\sigma}$-set. By (3.2), (3.9), and (3.11), we have $E_{1} \cap M\left(L^{\infty}\right)=\emptyset$. Hence by Lemma 3.1, $\overline{E_{1}} \cap M\left(L^{\infty}\right)=\emptyset$. Thus $x_{0} \notin \overline{E_{1}}$. By (3.11), $E=E_{1} \cup E_{2}$, so that by (3.7) we have

$$
x_{0} \in \overline{E_{2}} \subset\left\{\tilde{\chi}_{S_{1}}=1\right\} .
$$

Put

$$
V_{1}=\{f \neq 0\} \cap\left\{\tilde{\chi}_{S_{2}}<1\right\} \quad \text { and } \quad V_{2}=\{f \neq 0\} \cap\left\{\tilde{\chi}_{S_{2}}=1\right\} .
$$

Then $V_{1}$ is an $F_{\sigma}$-set, and by (3.9) $V_{1} \cap M\left(L^{\infty}\right)=\emptyset$. Hence by Lemma 3.1, $\bar{V}_{1} \cap M\left(L^{\infty}\right)=\emptyset$. Thus $x_{0} \notin \overline{V_{1}}$. By (3.13), $\{f \neq 0\}=V_{1} \cup V_{2}$. By (3.7), $x_{0} \in \overline{\{f \neq 0\}}$ so that

$$
x_{0} \in \overline{V_{2}} \subset\left\{\tilde{\chi}_{S_{2}}=1\right\} .
$$

By (3.1) and (3.10), we have $\left\{\tilde{\chi}_{S_{1}}=1\right\} \cap\left\{\tilde{\chi}_{S_{2}}=1\right\}=\emptyset$. But this contradicts (3.12) and (3.14). This completes the proof.

\section{Prime ideals in $H^{\infty}+C$}

The following theorem answers the problem posed by Gorkin and Mortini [7] Q4].

Theorem 4.1. For every $x \in M\left(H^{\infty}+C\right), J(x)$ is a prime ideal of $H^{\infty}+C$.

Proof. Let $f, g \in H^{\infty}+C$ such that $f g \in J(x)$. Since our topology of $M\left(H^{\infty}+C\right)$ is the weak ${ }^{*}$-topology, fundamental neighborhoods of $x$ are open $F_{\sigma}$-sets. Hence there is an open $F_{\sigma}$-subset $U$ of $M\left(H^{\infty}+C\right)$ such that $x \in U$ and

$$
f g=0 \text { on } U \text {. }
$$

To prove our theorem, suppose not; that is,

$$
f \notin J(x) \text { and } g \notin J(x) .
$$

If $f(x) \neq 0$, then by (4.1) we have $g \in J(x)$. So we may assume that

$$
f(x)=0 \text { and } g(x)=0 .
$$

Let

$$
U_{f}=U \cap\{f \neq 0\} \quad \text { and } \quad U_{g}=U \cap\{g \neq 0\} .
$$

Then by (4.2) and (4.3),

$$
x \in \overline{U_{f}} \cap \overline{U_{g}} .
$$

Since $U$ is an $F_{\sigma}$-set, $U_{f}$ is an $F_{\sigma}$-subset of $M\left(H^{\infty}+C\right)$, and by (4.1) and (4.4), $U_{f} \subset$ int $Z(g)$. Then by Theorem 3.1, $\overline{U_{f}} \subset$ int $Z(g)$. Since $U_{g} \subset\{g \neq 0\}$, we have $\overline{U_{f}} \cap \overline{U_{g}}=\emptyset$. This contradicts (4.5).

Let $x \in M\left(H^{\infty}+C\right)$. Then by Newman's theorem [14], $J(x) \cap H^{\infty}=\{0\}$ if and only if $x \in M\left(L^{\infty}\right)$. By Theorem 4.1, we have the following corollary.

Corollary 4.1. Let $x \in M\left(H^{\infty}+C\right) \backslash M\left(L^{\infty}\right)$. Then $J(x) \cap H^{\infty}$ is a prime ideal of $H^{\infty}$. 


\section{REFERENCES}

[1] L. Carleson, Interpolations by bounded analytic functions and the corona problem, Ann. of Math. 76(1962), 547-559. MR 25:5186

[2] T. W. Gamelin, Uniform Algebras, Prentice Hall, Englewood Cliffs, N.J., 1969. MR 53:14137

[3] J. Garnett, Bounded Analytic Functions, Academic Press, New York, 1981. MR 83g:30037

[4] P. Gorkin and R. Mortini, Interpolating Blaschke products and factorization in Douglas algebras, Michigan Math. J. 38(1991), 147-160. MR 92b:46083

[5] P. Gorkin and R. Mortini, Alling's conjecture on closed prime ideals in $H^{\infty}$, J. Funct. Anal. 148(1997), 185-190. MR 98d:46053

[6] P. Gorkin and R. Mortini, A survey of closed ideals in familiar function algebras, Function spaces (Edwardsville, IL, 1998), 161-170, Contemp. Math., 232, Amer. Math. Soc., Providence, RI, 1999. MR 2000b:46092

[7] P. Gorkin and R. Mortini, Synthesis sets for $H^{\infty}+C$, Indiana Univ. Math. J. 49 (2000), 287-309. CMP 2000:17

[8] C. Guillory, K. Izuchi and D. Sarason, Interpolating Blaschke products and division in Douglas algebras, Proc. Roy. Irish Acad. Sect.A 84(1984), 1-7. MR 86j:46054

[9] C. Guillory and D. Sarason, Division in $H^{\infty}+C$, Michigan Math. J. 28(1981), 173-181. MR 82j:46066

[10] K. Hoffman, Banach Spaces of Analytic Functions, Prentice Hall, Englewood Cliffs, N.J., 1962. MR 24:A2844

[11] K. Hoffman, Bounded analytic functions and Gleason parts, Ann. of Math. 86(1967), 74-111. MR 35:5945

[12] K. Izuchi, Factorization of Blaschke products, Michigan Math. J. 40(1993), 53-75. MR 94d:30062

[13] K. Izuchi, Weak infinite powers of Blaschke products, J. Anal. Math. 75(1998), 135-154. MR 99k:30061

[14] D. J. Newman, Some remarks on the maximal ideal space structure of $H^{\infty}$, Ann. of Math. 70(1959), 438-445. MR 21:5024

[15] C. Sundberg, A note on algebras between $L^{\infty}$ and $H^{\infty}$, Rocky Mount. J. Math. 11(1981), 333-336. MR 82g:46093

Department of Mathematics, Nitgata University, Nimgata 950-2181, Japan

E-mail address: izuchi@math.sc.niigata-u.ac.jp 